\title{
Dielectric Characterization and Multi-Stage Separation of Various Cells via Dielectrophoresis in Bipolar Electrode Arrayed Device
}

Tianyi Jiang ${ }^{1}$, Xiaoming Chen ${ }^{1}$, Yukun Ren ${ }^{* 1,2}$, Dewei Tang ${ }^{1}$, Hongyuan Jiang ${ }^{* 1}$

1. School of Mechatronics Engineering, Harbin Institute of Technology, Harbin 150001, PR China. Email: rykhit@hit.edu.cn

2. State Key Laboratory of Robotics and System, Harbin Institute of Technology, Harbin 150001, PR China. E-mail: jhy_hit@hit.edu.cn

Section 1. Theoretical basis

Section 2. Specific sizes of channel and BPEs

Section 3. Equations and conditions employed on the computational model

Section 4. Voltage effect on electric-field distribution

Section 5. Device configuration

Section 6. Performance of yeast cells in frequency-modulation electric field

Section 7. Focusing performance of silica particles

Section 8. Photographs of devices

Section 9. Voltage effect on separation of live and dead cells

Section 10. Initial states of microalgal cells

Section 11. Power consumption in the separation

Section 12. Release process of microalgal cells

Section 13. Standardization of microalga separation

Section 14. Supplementary Movies 


\section{Section 1. Theoretical basis}

\section{Induced charge electroosmosis}

When an AC signal $\phi=A \cos (\omega t+\theta)=\operatorname{Re}\left(A e^{j \theta} e^{j \omega t}\right)=\operatorname{Re}\left(\tilde{\phi} e^{j \omega t}\right)$ with an angular frequency $\omega=2 \pi f$ is applied on the left driving electrode, and the right driving electrode is grounded, an electric field is created in the fluid domain. If the phasor amplitude is introduced, the boundary conditions employed on the left and right driving electrodes are ${ }^{1}$ :

$$
\begin{gathered}
\tilde{\phi}=A e^{j \theta} \\
\tilde{\phi}=0
\end{gathered}
$$

Because the separation buffer is homogeneous-conductivity $\mathrm{KCl}$ electrolyte, the electrostatic potential in the fluid domain can be obtained by solving Laplace equation ${ }^{1}$ :

$$
\nabla^{2} \tilde{\phi}=0
$$

Under the driving of electric field, equal positive and negative ions migrate toward different sides of bipolar electrodes like charging process of a skin capacitance, forming induced double layer (IDL). IDL is equivalent to a combined capacitor by connecting stern layer and diffuse layer in series ${ }^{2}$.

$$
C_{E}=\frac{C_{D} C_{S}}{C_{D}+C_{S}}=\frac{1}{1+\delta} C_{D}
$$

where, $C_{D}$ and $C_{s}$ indicate capacitance of diffuse layer and stern layer, respectively; $\delta$ is the ratio of diffuse layer and stern layer capacitance: $\delta=C_{D} / C_{S}$.

In general, electrolyte and IDL above the floating electrode are regarded as resistance and capacitance, and they are connected serially. In this way, we can investigate charging process of IDL as RC circuit. After RC characteristic time $\tau_{R C}=R C_{D} / \sigma=\lambda_{d} R / D$, IDL reaches a steady state due to the balance between electrostatic attraction and thermal diffusion. In this scenario, Zeta potential across the diffuse layer can be calculated by ${ }^{1}$ :

$$
\zeta=\frac{1}{1+\delta}\left(\tilde{\phi}_{0}-\tilde{\phi}\right)
$$

For the diffuse layer, the charge-voltage relationship can be obtained ${ }^{3,4}$

$$
q=C_{E}\left(\tilde{\phi}_{0}-\tilde{\phi}\right)=\frac{1}{1+\delta} C_{E}\left(\tilde{\phi}_{0}-\tilde{\phi}\right)=C_{E} \zeta
$$

The normal current continuity can elucidate conservation of diffuse charge in the IDL and describe this interfacial jump condition ${ }^{1}$.

$$
\sigma_{m} \cdot \boldsymbol{n} \nabla \phi=C_{E} \frac{\partial\left(\tilde{\phi}_{b}-\tilde{\phi}_{0}\right)}{\partial t}
$$

where, $\tilde{\phi}_{b}$ is the potential in the fluid domain, $\tilde{\phi}_{0}$ stands for the potential at the gate electrode, $n$ represents unit normal vector. Meanwhile, the channel walls were employed following boundary conditions $^{3-5}$ :

$$
\boldsymbol{n} \cdot \nabla \tilde{\phi}=0
$$


In this scenario, no normal electric-field can penetrate IDL, and tangential electric-field drives excessive counterions within the diffuse layer to move along or against the electric field.

$$
\tilde{\boldsymbol{E}}_{\boldsymbol{t}}=\tilde{\boldsymbol{E}}-\tilde{\boldsymbol{E}} \cdot \boldsymbol{n} \cdot \boldsymbol{n}
$$

If $1 / 2 \pi f$ is longer than the charging time of IDL, based on Helmholtz-Smoluchowski formula induced charge electroosmotic (ICEO) flow on the bipolar electrodes can be described as ${ }^{3,5}$ :

$$
\begin{aligned}
\left\langle\boldsymbol{u}_{E O F}\right\rangle & =-\frac{\varepsilon_{m}\left\langle\zeta \boldsymbol{E}_{\boldsymbol{t}}\right\rangle}{\eta}=-\frac{\varepsilon_{m}\left\langle\tilde{\zeta} \tilde{\boldsymbol{E}}_{t}^{*}\right\rangle}{\eta} \\
& =\frac{\varepsilon_{m}}{2 \eta(1+\delta)} \operatorname{Re}\left[\left(\tilde{\phi}_{b}-\tilde{\phi}_{0}\right)(\tilde{\boldsymbol{E}}-\tilde{\boldsymbol{E}} \cdot \boldsymbol{n} \cdot \boldsymbol{n})^{*}\right]
\end{aligned}
$$

where, $\varepsilon_{m}$ indicates the permittivity of fluid. $\eta$ represents dynamic viscosity of fluid.

Equation (S10) as the boundary condition is employed on the ends of BPEs. Besides, the slip velocities are employed on the left and right electrodes due to the action of $\mathrm{AC}$ electroosmosis:

$$
\begin{aligned}
& \left\langle\boldsymbol{u}_{L}\right\rangle=\frac{\varepsilon_{m}}{2 \eta(1+\delta)} \operatorname{Re}\left[(\tilde{\phi}-A)(\tilde{\boldsymbol{E}}-\tilde{\boldsymbol{E}} \cdot \boldsymbol{n} \cdot \boldsymbol{n})^{*}\right] \\
& \left\langle\boldsymbol{u}_{R}\right\rangle=\frac{\varepsilon_{m}}{2 \eta(1+\delta)} \operatorname{Re}\left[\tilde{\phi}(\tilde{\boldsymbol{E}}-\tilde{\boldsymbol{E}} \cdot \boldsymbol{n} \cdot \boldsymbol{n})^{*}\right]
\end{aligned}
$$

Besides, the rest channel walls are defined as no slip walls ${ }^{1}$ :

$$
\boldsymbol{u}=0
$$

Moreover, the fluid domain is governed by the full Stokes equation ${ }^{1}$.

$$
\nabla \boldsymbol{u}=0
$$

$$
\rho_{\mathrm{m}}\left[\frac{\partial \boldsymbol{u}}{\partial t}+\boldsymbol{u} \nabla \boldsymbol{u}\right]=-\nabla p+\nabla\left[\eta\left(\nabla \boldsymbol{u}+(\nabla \boldsymbol{u})^{T}\right)\right]+\boldsymbol{f}
$$

In this scenario, we can obtain the distribution of fluid velocity in the channel caused by ICEO flow.

\section{Dielectrophoresis}

When a particle is suspended in the electrolyte, the interaction between particle and electric field produces an effective dipole moment of particle. In the uniform electric field, there is no movement due to equal and opposite forces on the two poles. If the electric field is non-uniform, particles will move due to different force on the two poles. In the process, the polarized particle is equivalent to a dipole, and different poles experience different forces in the non-uniform electric field. The combined force of the particle is ${ }^{6}$

$$
\boldsymbol{F}=Q \boldsymbol{E}(r+d)-Q \boldsymbol{E}(r)
$$

If we expand the non-uniform electric field $\boldsymbol{E}$ around $r$ using Taylor series, the combined force on the particle can be shown $a^{7}$ :

$$
\boldsymbol{F}=Q \boldsymbol{E}(\boldsymbol{r})+Q\left(d x \frac{\partial}{\partial x}+d y \frac{\partial}{\partial y}+d z \frac{\partial}{\partial z}\right) \boldsymbol{E}+\text { higher order terms }-Q \boldsymbol{E}(\boldsymbol{r})
$$

By neglecting the higher-order terms, we can get the DEP force expression on the dipole ${ }^{7}$

$$
\boldsymbol{F}_{D E P}=(\boldsymbol{p} \cdot \nabla) \boldsymbol{E}
$$


In the AC electric field, the dipole moment of the particle can be expressed as:

$$
\begin{gathered}
\tilde{\boldsymbol{p}}=v \alpha \boldsymbol{E} e^{j \omega t} \\
\left\langle\boldsymbol{F}_{D E P}\right\rangle=\frac{1}{2} \operatorname{Re}\left[(\tilde{\boldsymbol{p}} \cdot \nabla) \boldsymbol{E}^{*}\right]=\frac{1}{2} v \operatorname{Re}[\tilde{\alpha}](\boldsymbol{E} \cdot \nabla) \boldsymbol{E}
\end{gathered}
$$

Substituting $\nabla(\boldsymbol{E} \cdot \boldsymbol{E})=2(\boldsymbol{E} \cdot \nabla) \boldsymbol{E}+2 \boldsymbol{E} \times(\nabla \times \boldsymbol{E})$ and $\nabla \times \boldsymbol{E}=0$ into equation (S20), the DEP force expression can be rewritten as ${ }^{7}$ :

$$
\left\langle\boldsymbol{F}_{D E P}\right\rangle=\frac{1}{4} v \operatorname{Re}[\tilde{\alpha}] \nabla(\boldsymbol{E} \cdot \boldsymbol{E})=\frac{1}{4} v \operatorname{Re}[\tilde{\alpha}] \nabla|\boldsymbol{E}|^{2}
$$

If a particle is suspended in a homogenous electrolyte, the potentials inside and outside the particle are ${ }^{7}$ :

$$
\left\{\begin{array}{l}
\phi_{m}=\left[\left(\frac{\tilde{\varepsilon}_{p}-\tilde{\varepsilon}_{m}}{\tilde{\varepsilon}_{p}+2 \tilde{\varepsilon}_{m}}\right) \frac{a^{3}}{r^{3}}-1\right] \operatorname{Er} \cos \theta \\
\phi_{p}=-\left(\frac{3 \tilde{\varepsilon}_{m}}{\tilde{\varepsilon}_{p}+2 \tilde{\varepsilon}_{m}}\right) E r \cos \theta
\end{array}\right.
$$

where, $\tilde{\varepsilon}_{m}$ indicates the complex permittivity of fluid. $\tilde{\varepsilon}_{p}$ represents complex permittivity of particles.

The outside potential of particle can be rewritten as $^{7}$ :

$$
\phi_{m}=\left(\frac{\tilde{\varepsilon}_{p}-\tilde{\varepsilon}_{m}}{\tilde{\varepsilon}_{p}+2 \tilde{\varepsilon}_{m}}\right) \frac{E a^{3}}{r^{2}} \cos \theta-E r \cos \theta
$$

At the right-hand side, the first term is the potential of dipole moment, which can be written as ${ }^{7}$ :

$$
\begin{gathered}
\boldsymbol{p}=4 \pi \varepsilon_{m}\left(\frac{\tilde{\varepsilon}_{p}-\tilde{\varepsilon}_{m}}{\tilde{\varepsilon}_{p}+2 \tilde{\varepsilon}_{m}}\right) a^{3} \boldsymbol{E} \\
\boldsymbol{p}=v \tilde{\alpha} \boldsymbol{E}=\frac{4 \pi a^{3}}{3} \tilde{\alpha} \boldsymbol{E}
\end{gathered}
$$

According to equation (S24) and (25), the effective polarizability is ${ }^{7}$ :

$$
\begin{gathered}
\tilde{\alpha}=3 \varepsilon_{m}\left(\frac{\tilde{\varepsilon}_{p}-\tilde{\varepsilon}_{m}}{\tilde{\varepsilon}_{p}+2 \tilde{\varepsilon}_{m}}\right)=3 \varepsilon_{m} \tilde{f}_{C M} \\
\tilde{f}_{C M}=\frac{\tilde{\varepsilon}_{p}-\tilde{\varepsilon}_{m}}{\tilde{\varepsilon}_{p}+2 \tilde{\varepsilon}_{m}}
\end{gathered}
$$

We often use a spherical particle with a single shell to investigate the biological particles, involving cells and viruses. In this case, effective polarizability is ${ }^{8}$ :

$$
\begin{gathered}
\tilde{\alpha}=3 \varepsilon_{1}\left(\frac{\tilde{\varepsilon}_{23}-\tilde{\varepsilon}_{1}}{\tilde{\varepsilon}_{23}+2 \tilde{\varepsilon}_{1}}\right) \\
\tilde{\varepsilon}_{23}=\tilde{\varepsilon}_{2}\left[\left(\frac{a_{1}}{a_{2}}\right)^{3}+2\left(\frac{\tilde{\varepsilon}_{3}-\tilde{\varepsilon}_{2}}{\tilde{\varepsilon}_{3}+2 \tilde{\varepsilon}_{2}}\right)\right] /\left[\left(\frac{a_{1}}{a_{2}}\right)^{3}-\left(\frac{\tilde{\varepsilon}_{3}-\tilde{\varepsilon}_{2}}{\tilde{\varepsilon}_{3}+2 \tilde{\varepsilon}_{2}}\right)\right]
\end{gathered}
$$


where, $\quad \tilde{\varepsilon}_{1}=\varepsilon_{1}-j \sigma_{1} /(2 \pi f), \quad \tilde{\varepsilon}_{2}=\varepsilon_{2}-j \sigma_{2} /(2 \pi f), \quad \tilde{\varepsilon}_{3}=\varepsilon_{3}-j \sigma_{3} /(2 \pi f), \gamma_{12}=a_{1} / a_{2} . \quad \varepsilon_{1}$, and $\sigma_{1}$ indicate the permittivity and conductivity of separation buffer; $\varepsilon_{2}$ and $\sigma_{2}$ are the permittivity and conductivity of cell membrane; $\varepsilon_{3}$ and $\sigma_{3}$ stand for the permittivity and conductivity of cell core; $a_{1}$ and $a_{2}$ indicate the size of cell core and cell membrane; $f$ is the voltage frequency.

Substituting effective polarizability into equation (S20), we can obtain the well-known expression ${ }^{9,10}$ :

$$
\left\langle\boldsymbol{F}_{D E P}\right\rangle=\pi \varepsilon_{m} a^{3} \operatorname{Re}\left[\tilde{f}_{C M}\right] \nabla|\boldsymbol{E}|^{2}=\pi \varepsilon_{m} a^{3} \operatorname{Re}\left[\frac{\tilde{\varepsilon}_{p}-\tilde{\varepsilon}_{m}}{\tilde{\varepsilon}_{p}+2 \tilde{\varepsilon}_{m}}\right] \nabla|\boldsymbol{E}|^{2}
$$

If $\operatorname{Re}\left[\tilde{f}_{C M}\right]>0$, particles experience positive DEP force, and they are attracted to the high electric field region. If $\operatorname{Re}\left[\tilde{f}_{C M}\right]<0$, particles experience negative DEP force, and they are repelled away from the high electric field region.

\section{Alternating current electrothermal}

Under the effect of AC electric field, Joule heating in the separation buffer leads to a temperature rise. Consequently, the property of separation buffer becomes nonuniform for conductivity and permittivity liner change ${ }^{11,12}$ :

$$
\begin{gathered}
\varepsilon(T)=\varepsilon\left(T_{0}\right)\left(1+\alpha\left(T-T_{0}\right)\right) \\
\sigma(T)=\sigma\left(T_{0}\right)\left(1+\beta\left(T-T_{0}\right)\right)
\end{gathered}
$$

In the $\mathrm{AC}$ electric field, the charge conservation equation can be written as ${ }^{12}$ :

$$
\begin{aligned}
& \nabla((\sigma(T)+j \omega \varepsilon(T)) \boldsymbol{E})=0 \\
& \Rightarrow \nabla \cdot \boldsymbol{E}=-\frac{\nabla \sigma(T)+j \omega \nabla \varepsilon(T)}{\sigma(T)+j \omega \varepsilon(T)} \boldsymbol{E}
\end{aligned}
$$

Substituting equation (R31) into equation (R32)

$$
\nabla^{2} \phi=\gamma \cdot \nabla \phi=\left(-\frac{\sigma\left(T_{0}\right) \beta+j \omega \varepsilon\left(T_{0}\right) \alpha}{\sigma(T) \beta+j \omega \varepsilon(T)} \nabla T\right) \cdot \nabla \phi
$$

Based on Gauss law, we can obtain the charge density:

$$
\rho_{f}=\nabla \cdot(\varepsilon(T) \boldsymbol{E})=\frac{(\sigma(T) \nabla \varepsilon(T)-\varepsilon(T) \nabla \sigma(T))}{\sigma(T)+j \omega \varepsilon(T)} \cdot \boldsymbol{E}
$$

In this scenario, there is a dielectric gradient in the buffer, and the electric field exerts a volume force on the fluid. Bulk electro-convection takes place in the channel. Volume force density exerting on the fluid is ${ }^{12}$ : 


$$
\boldsymbol{f}_{E}=\rho E-\frac{1}{2}|\boldsymbol{E}|^{2} \nabla \varepsilon+\nabla\left[\frac{1}{2}|\boldsymbol{E}|^{2}\left(\frac{\partial \varepsilon}{\partial \rho_{m}}\right) \rho_{m}\right]
$$

Time-averaged electrothermal force per unit volume, which can be expressed as

$$
\left\langle\boldsymbol{f}_{E T}\right\rangle=\frac{1}{2} \operatorname{Re}\left(\frac{\sigma(T) \varepsilon\left(T_{0}\right) \alpha-\varepsilon(T) \sigma\left(T_{0}\right) \beta}{\sigma(T)+j \omega(T)} \nabla T \cdot \boldsymbol{E} \cdot \boldsymbol{E}^{*}-\frac{1}{2}\left(\boldsymbol{E} \cdot \boldsymbol{E}^{*}\right) \varepsilon\left(T_{0}\right) \alpha \nabla T\right)
$$

where, $\alpha=\frac{\partial \varepsilon}{\partial T}=-0.004\left(K^{-1}\right), \quad \beta=\frac{\partial \sigma}{\partial T}=-0.02\left(K^{-1}\right)$

The governed equation employed in the fluid domain can be rewritten as:

$$
\rho_{\mathrm{m}}\left[\frac{\partial \boldsymbol{u}}{\partial t}+\boldsymbol{u} \nabla \boldsymbol{u}\right]=-\nabla p+\nabla\left[\eta\left(\nabla \boldsymbol{u}+(\nabla \boldsymbol{u})^{T}\right)\right]+\left\langle\boldsymbol{f}_{E T}\right\rangle
$$

In the PDMS channel, ITO electrode, and fluid domain, the temperature field is computed by solving the following equation ${ }^{12}$ :

$$
\rho_{m} c_{p}\left(\frac{\partial T}{\partial t}+(\boldsymbol{u} \cdot \nabla) T\right)=\nabla\left(k_{m} \nabla T\right)+\frac{1}{2} \sigma(T)\left(\boldsymbol{E} \cdot \boldsymbol{E}^{*}\right)
$$

where, $c_{p}$ indicates heat capacity; $k_{m}$ stands for thermal conductivity.

The boundary conditions employed on the electrode, fluid and PDMS are ${ }^{13,14}$ :

$$
\begin{gathered}
\rho c_{e} u \nabla T-\nabla\left(k_{e} \nabla T\right)=0 \\
\rho c_{f} u \nabla T-\nabla\left(k_{f} \nabla T\right)=\frac{1}{2} \sigma E \\
\rho c_{p} u \nabla T-\nabla\left(k_{p} \nabla T\right)=0
\end{gathered}
$$

where, $c_{e} / c_{f} / c_{p}$ indicates heat capacity of electrode/ fluid/ PDMS; $k_{e} / k_{f} / k_{p}$ stands for thermal conductivity of electrode/ fluid/ PDMS.

Temperatures on the PDMS/air interface and glass/air interface are set to room temperature, 293.15 K. 
Section 2. Specific sizes of channel and BPEs

Table S1 Specific sizes of channel and BPEs

\begin{tabular}{|c|c|c|c|c|c|c|}
\hline Parameter & $L_{1}$ & $L_{2}$ & $L_{3}$ & $L_{4}$ & $L_{5}$ & $H$ \\
\hline Value $(\mu \mathrm{m})$ & 100 & 200 & 75 & 75 & 150 & 76 \\
\hline
\end{tabular}




\section{Section 3. Equations and conditions employed on the computational model}

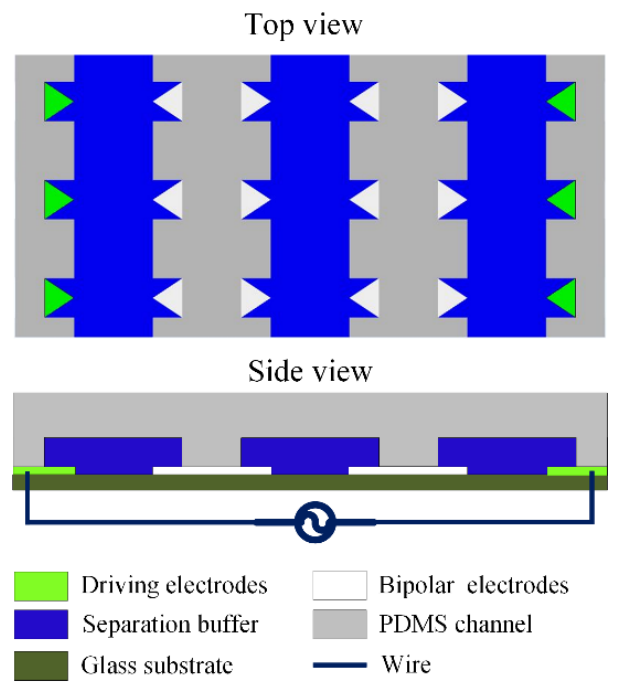

Figure S1 Configuration of the computational model.

Table S2 Equations and boundary conditions employed on the computational model

\begin{tabular}{|c|c|c|c|c|c|c|c|}
\hline Position & $\begin{array}{c}\text { Left } \\
\text { driving } \\
\text { electrode }\end{array}$ & $\begin{array}{c}\text { Right } \\
\text { driving } \\
\text { electrode }\end{array}$ & $\begin{array}{c}\text { Bipolar } \\
\text { electrode }\end{array}$ & $\begin{array}{c}\text { Separation } \\
\text { buffer }\end{array}$ & $\begin{array}{c}\text { PDMS } \\
\text { wall }\end{array}$ & $\begin{array}{c}\text { PDMS-air } \\
\text { interface }\end{array}$ & $\begin{array}{c}\text { Glass-air } \\
\text { interface }\end{array}$ \\
\hline Electric field & $(\mathrm{S} 1)$ & $(\mathrm{S} 2)$ & $(\mathrm{S} 7)$ & $(\mathrm{S} 3)$ & $(\mathrm{S} 8)$ & & - \\
\hline Flow field & $(\mathrm{S} 11)$ & $(\mathrm{S} 12)$ & $(\mathrm{S} 10)$ & $(\mathrm{S} 37)$ & $(\mathrm{S} 13)$ & & - \\
\hline $\begin{array}{c}\text { Temperature } \\
\text { field }\end{array}$ & $(\mathrm{S} 39)$ & $(\mathrm{S} 39)$ & $(\mathrm{S} 39)$ & $(\mathrm{S} 40)$ & $(\mathrm{S} 41)$ & $\mathrm{T}=293.15 \mathrm{~K}$ & $\mathrm{~T}=293.15 \mathrm{~K}$ \\
\hline
\end{tabular}




\section{Section 4. Voltage effect on electric-field distribution}
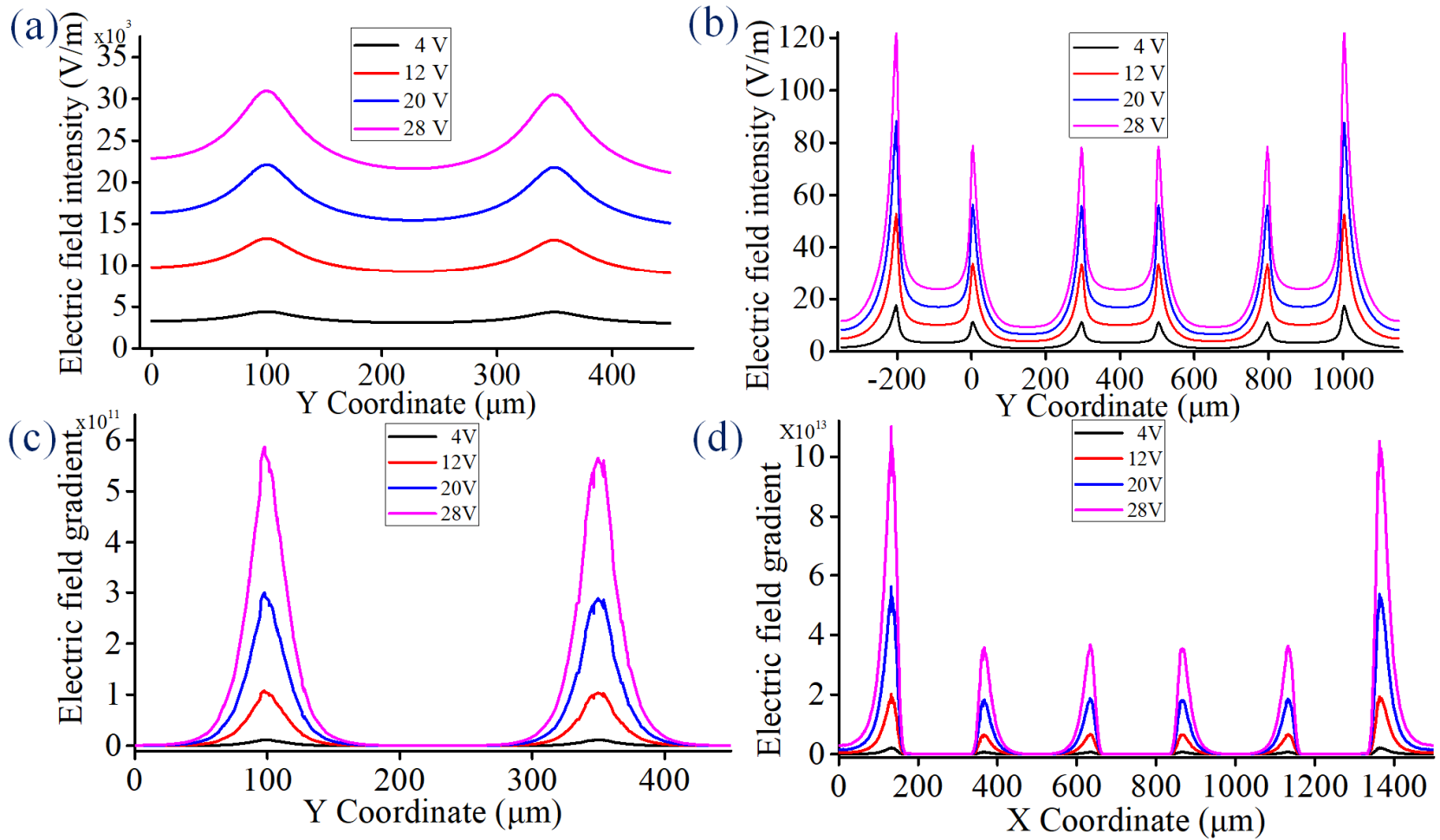

Figure S2 Voltage effect on distributions of flow and electric fields. (a) and (b) Influence of voltage intensity on electric field strength along cutting line 1 and 2. (c) and (d) Effect of voltage on the electric field gradient along cutting line 1 and 2 . 
Section 5. Device configuration

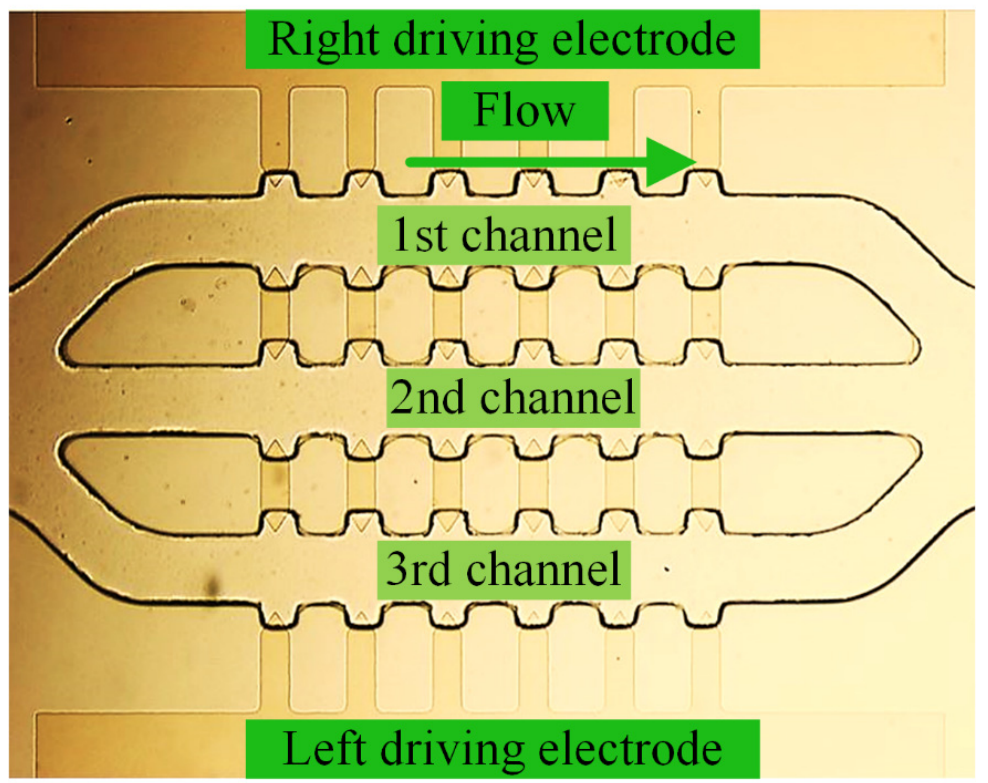

Figure S3 Channel and electrode configuration. 
Section 6. Performance of yeast cells in frequency-modulation electric field
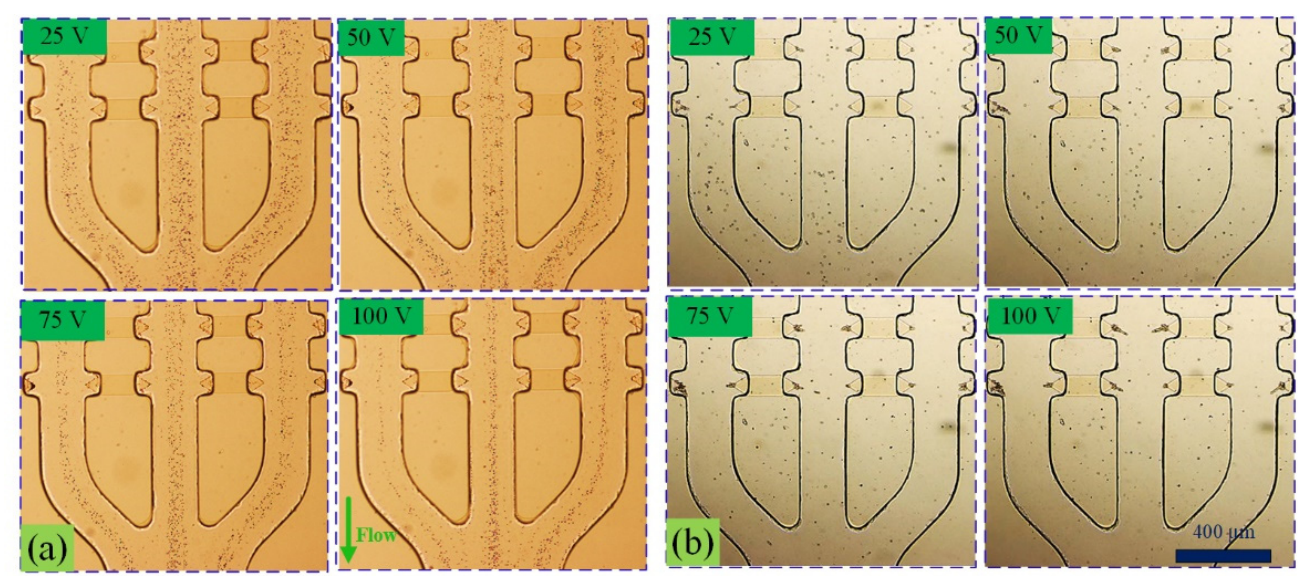

Figure S4 Experimental images illustrating DEP response of microalgal cells. Experiment images illustrating (a) focusing and (b) capturing of yeast cells. 


\section{Section 7. Focusing performance of silica particles}


Figure S5 Focusing width of silica particles in 3-channel device. (a) Focusing width against voltage. (b) Experiment images illustrating focusing of silica particle 
Section 8. Photographs of devices
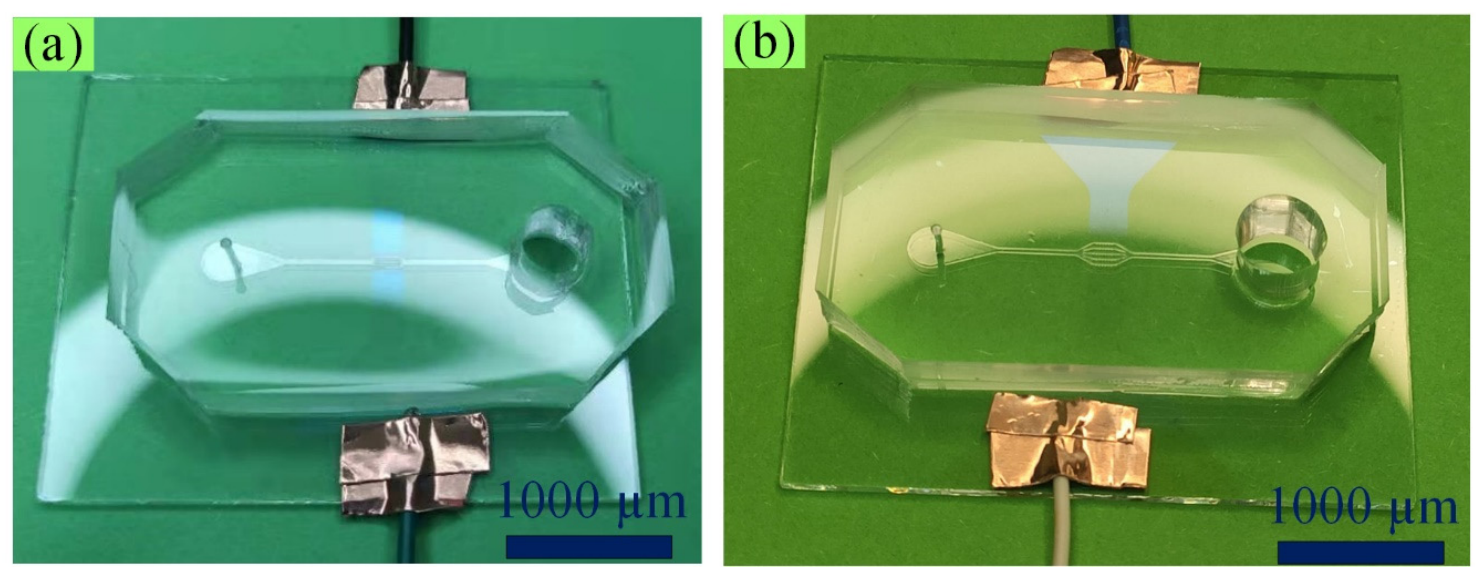

Figure S6 Photographs of devices. (a) 3-channel device. (b) 5-channel device. 
Section 9. Voltage effect on separation of live and dead cells

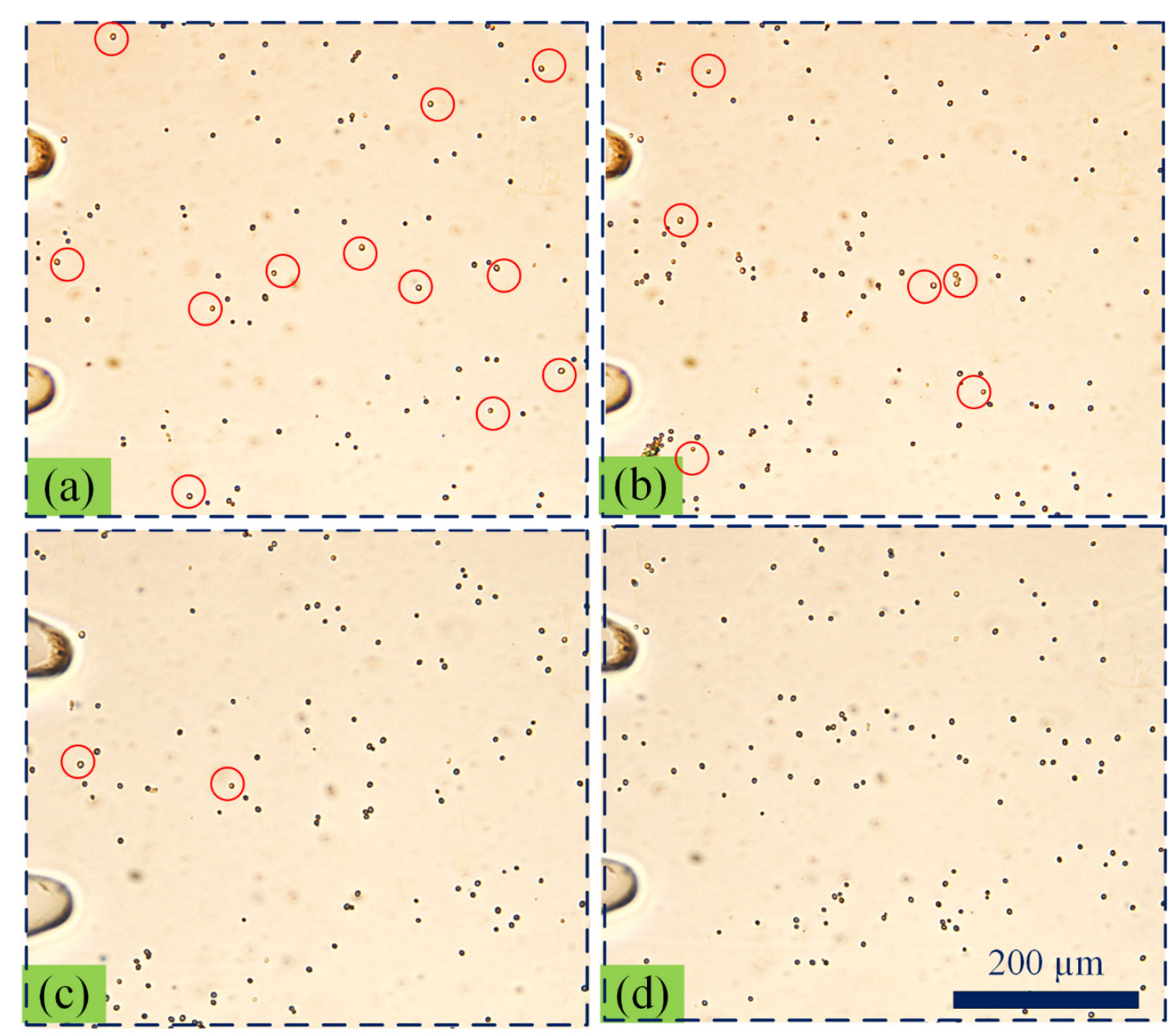

Figure S7 Experimental images demonstrating the separation results under different voltages. (a) A=150 V. (b) 175 V. (c) $200 \mathrm{~V}$. (d) $250 \mathrm{~V}$. Live cells are marked by red circles 
Section 10. Initial states of microalgal cells

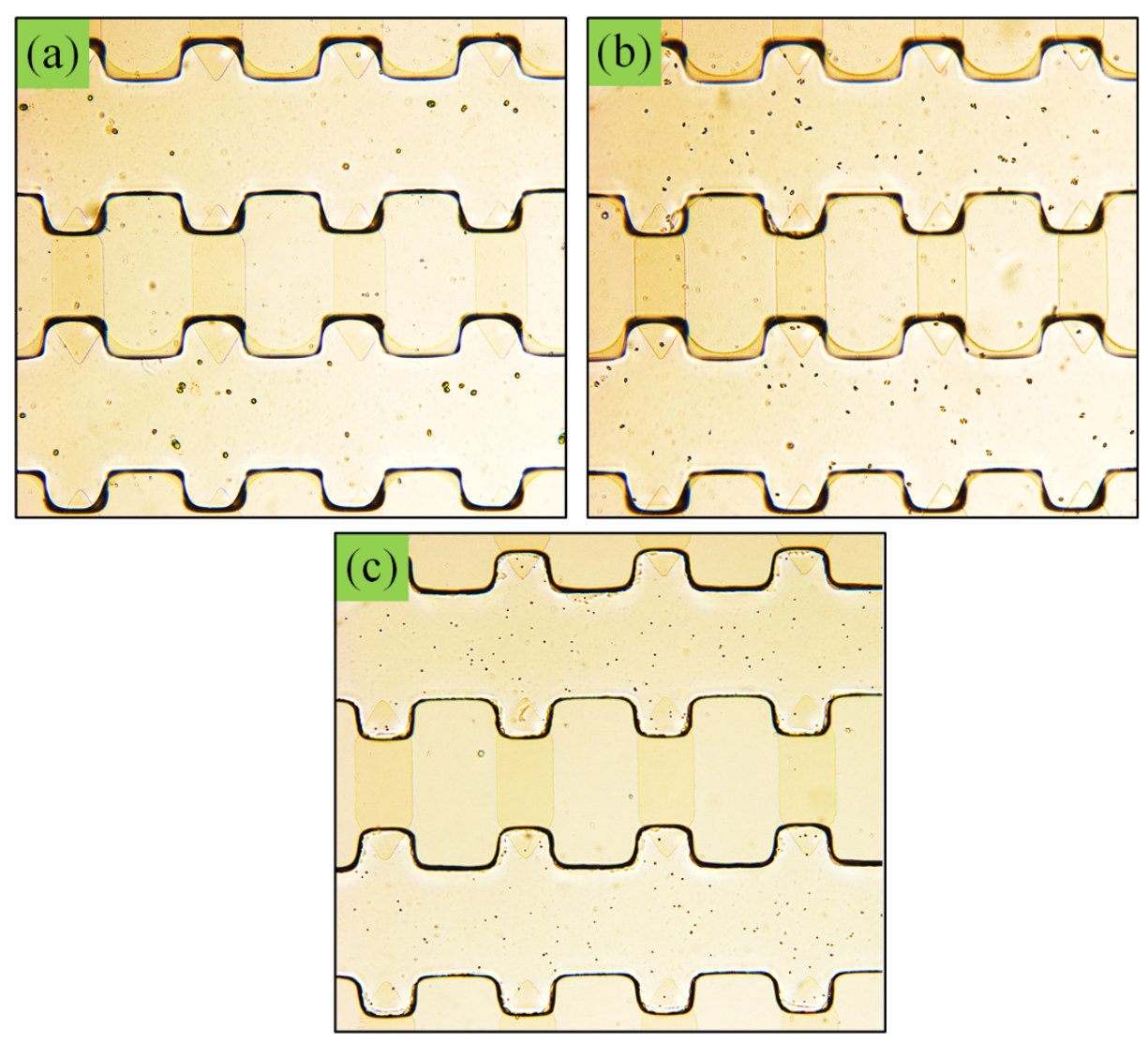

Figure S8 Initial states of different microalgal cells in the branching channels. (a) Oocystis sp. (b) Scenedesmus sp. (c) C. vulgaris. 


\section{Section 11. Power consumption in the separation}

To calculate the power consumed in the separation, we measured the electric current under different voltage amplitude at the frequency of $3 \mathrm{kHz}$, and conductivity of $1 \mathrm{mS} / \mathrm{m}$. Plot illustrating the relationship between electric current and voltage amplitude is give Fig.S9.

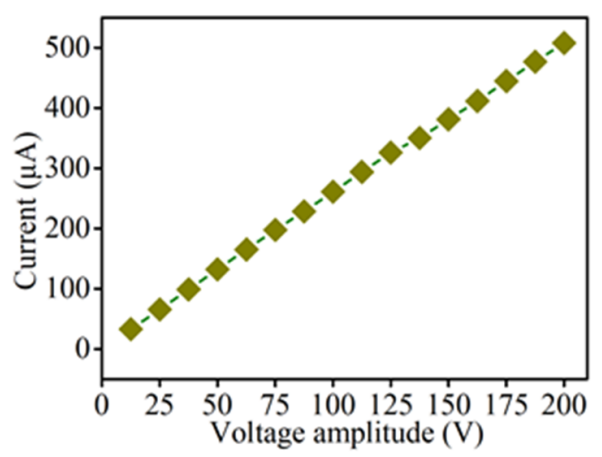

Figure S9 Relationship between current and voltage amplitude

In the isolation process of $C$. vulgaris, the voltage amplitude is about $87.5 \mathrm{~V}$, and electric current measured in experiment is about $0.2283 \mathrm{~mA}=2.283 \times 10^{-4} \mathrm{~A}$ (Fig.S9). The electrical energy consumed in the separation of $C$. vulgaris per hour is

$$
P=U I=\frac{87.5}{\sqrt{2}} \times 2.2832 \times 10^{-4} \approx 1.41 \times 10^{-2}[\mathrm{~W}]
$$




\section{Section 12. Release process of microalgal cells}
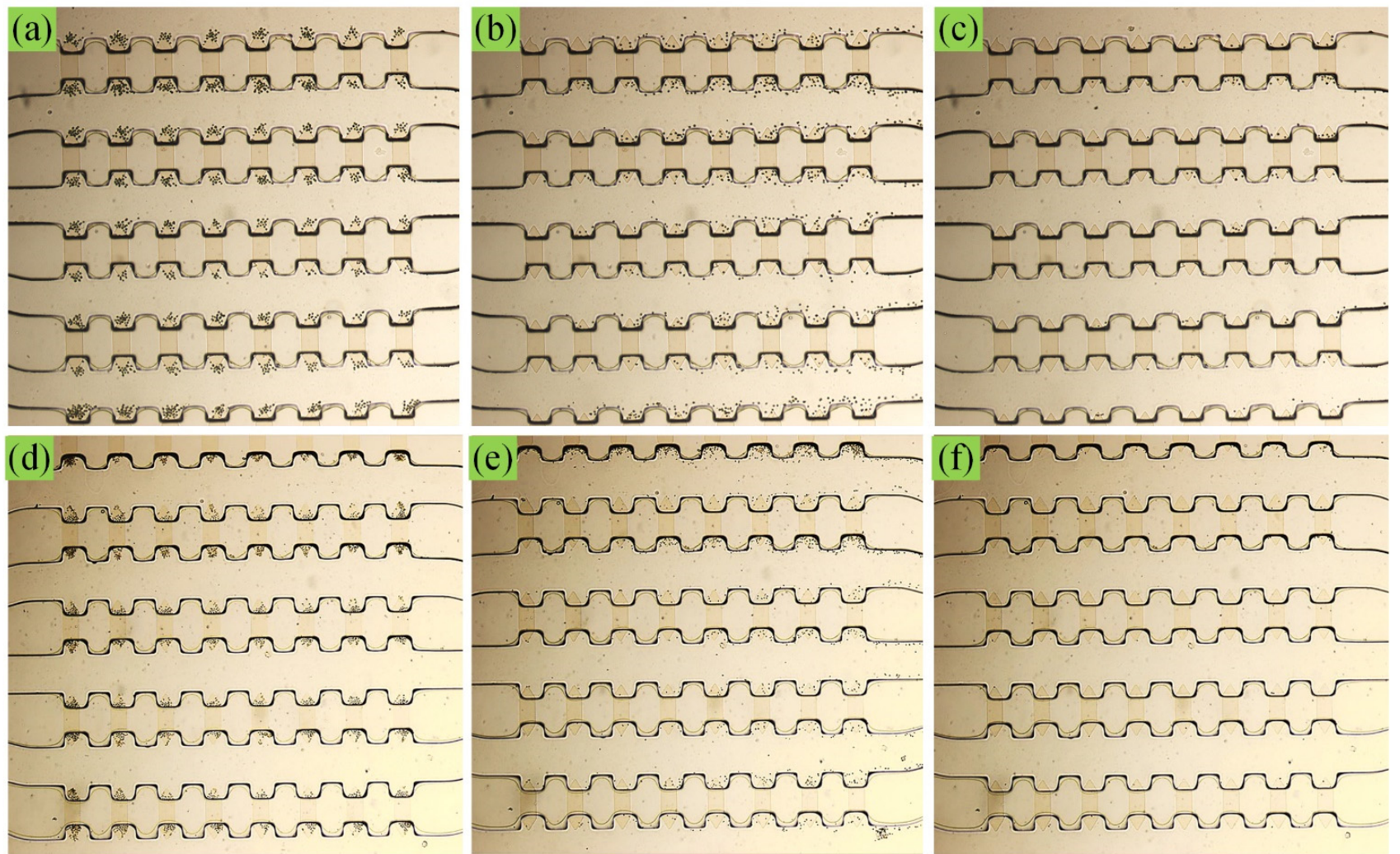

Figure S10 Experimental images demonstrating the separation results under different voltages. (a-c)

Oocystis sp. (d-f) Scenedesmus sp. 


\section{Section 13. Standardization of microalga separation}

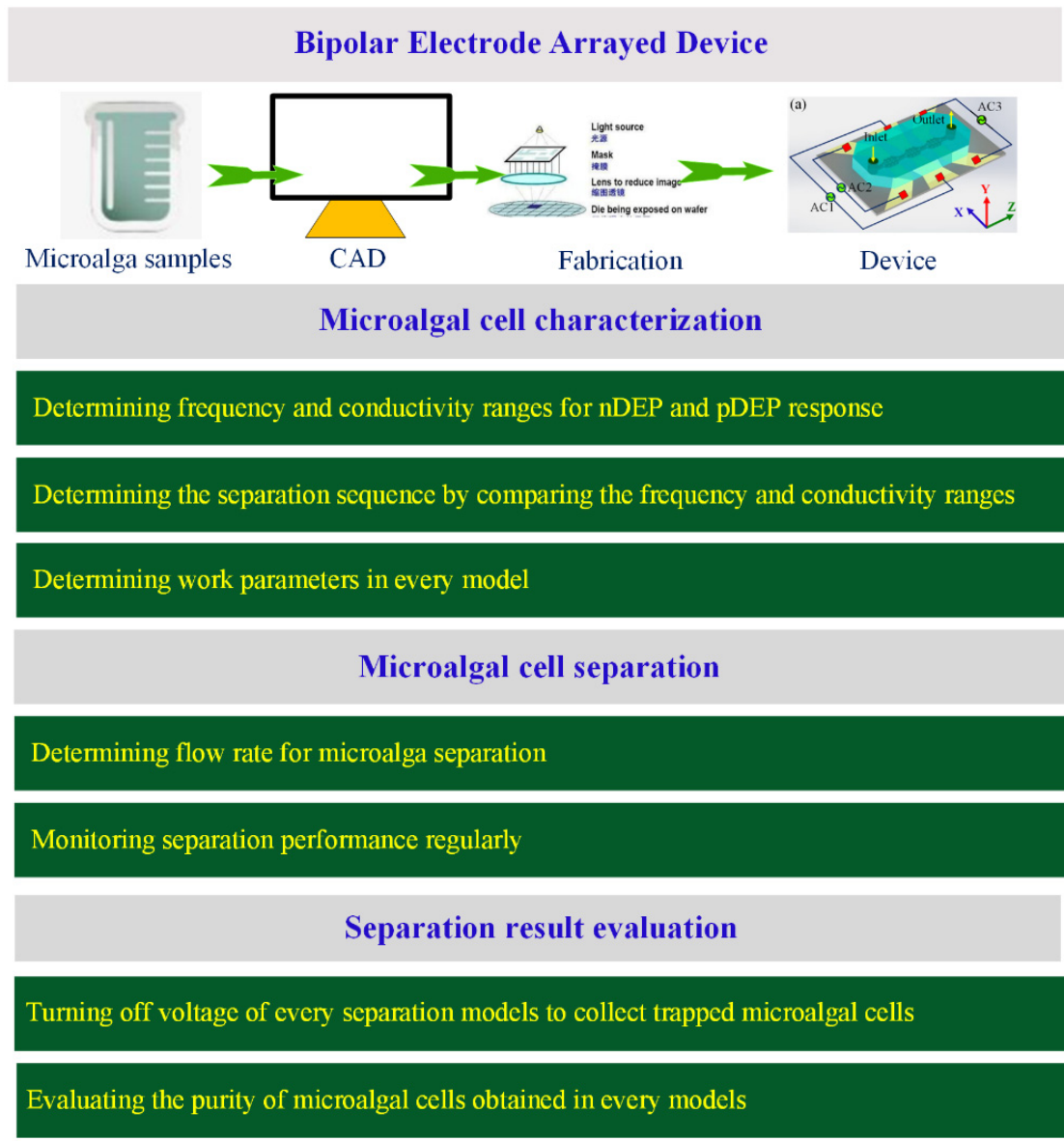

Figure S11 Standardization of microalga separation in the bipolar electrode arrayed device.

In the first place, we need to determine the channel number and separation models according to the cell species and sample volume. We design the device through the CAD and fabricate desired separation device through the standard soft lithography technique;

In the second place, microalgal cells need to be characterized to determine the frequency and conductivity ranges for their nDEP and pDEP responses. According to their DEP responses, we can determine the buffer conductivity, separation sequence, and specific work parameters for their separation;

In the third place, we introduce the microalgal samples into the separation device. According to the separation performance of every model, we can determine the flow rate. In the separation process, we need to monitor the separation performance every 20 mins.

In the fourth place, when all the samples are processed, voltages on every model need to be switched off to release trapped microalgal cells orderly. Then, purity of microalgal cells are calculated to evaluate the separation performance. 


\section{Section 14. Supplementary Movies}

Video S1: Separation of silica particles and yeast cells in 3- and 5-channel devices.

Video S2: Separation of live and dead cells in 3- and 5-channel devices.

Video S3: Separation of Oocystis sp. and C. vulgaris.

Video S4: Sequential separation of Oocystis sp., Scenedesmus sp., C. vulgaris in multi-stage separation device.

Video S5: Release processes of Oocystis sp. and Scenedesmus sp. 


\section{References}

(1) Ren, Y.; Liu, W.; Jia, Y.; Tao, Y.; Shao, J.; Ding, Y.; Jiang, H., Induced-charge electroosmotic trapping of particles, Lab on a chip 2015, 15, 2181-2191.

(2) Squires, T. M.; Bazant, M. Z., Induced-charge electro-osmosis, Journal of Fluid Mechanics 2004, 509, $217-252$.

(3) Peng, C.; Lazo, I.; Shiyanovskii, S. V.; Lavrentovich, O. D., Induced-charge electro-osmosis around metal and Janus spheres in water: Patterns of flow and breaking symmetries, Physical review. E, Statistical, nonlinear, and soft matter physics 2014, 90 , 051002.

(4) Ren, Y.; Liu, W.; Liu, J.; Tao, Y.; Guo, Y.; H Jiang., Particle rotational trapping on a floating electrode by rotating inducedcharge electroosmosis, Biomicrofluidics, 2016, 10, 054103.

(5) Pascall, A. J.; Squires, T. M., Induced charge electro-osmosis over controllably contaminated electrodes, Physical review letters 2010, 104, 088301.

(6) Hu, X.; Bessette, P. H.; Qian, J.; Meinhart, C. D.; Daugherty, P. S.; Soh, H. T., Marker-specific sorting of rare cells using dielectrophoresis, Proceedings of the National Academy of Sciences of the United States of America 2005, 102, 15757-15761.

(7) H Morgan, NG Green., AC electrokinetics: colloids and nanoparticles, Research Studies Press 2003.

(8) Yan, S.; Zhang, J.; Li, M.; Alici, G.; Du, H.; Sluyter, R.; Li, W., On-chip high-throughput manipulation of particles in a dielectrophoresis-active hydrophoretic focuser, Scientific reports 2014, 4, 5060.

(9) Zhao, K.; Larasati; Duncker, B. P.; Li, D., Continuous cell characterization and separation by microfluidic alternating current dielectrophoresis, Analytical chemistry 2019, 91, 6304-6314.

(10) Zhao, K.; Peng, R.; Li, D., Separation of nanoparticles by a nano-orifice based DC-dielectrophoresis method in a pressuredriven flow, Nanoscale 2016, 8, 18945-18955.

(11) Liu, W.; Ren, Y.; Tao, Y.; Yao, B.; Li, Y., Simulation analysis of rectifying microfluidic mixing with field-effect-tunable electrothermal induced flow, Electrophoresis 2018, 39, 779-793.

(12) Malekanfard, A.; Liu, Z.; Song, L.; Kale, A.; Zhang, C.; Yu, L.; Song, Y.; Xuan, X., Joule heating-enabled electrothermal enrichment of nanoparticles in insulator-based dielectrophoretic microdevices, Electrophoresis 2021, 42, 626-634.

(13) Zhang, K.; Ren, Y.; Zhao, M.; Jiang, T.; Hou, L.; Jiang, H., Flexible Microswimmer Manipulation in Multiple Microfluidic Systems Utilizing Thermal Buoyancy-Capillary Convection, Analytical chemistry 2021, 93, 2560-2569.

(14) Zhang, K.; Ren, Y.; Hou, L.; Jiang, T.; Jiang, H., Flexible Particle Focusing and Switching in Continuous Flow via Controllable Thermal Buoyancy Convection, Analytical chemistry 2020, 92, 2778-2786. 